livraisons

d'Histoire

de l'Architecture

\section{Livraisons de l'histoire de l'architecture}

$18 \mid 2009$

D'architectures

\title{
Henri-Marcel Magne (1877-1944) \\ Henri-Marcel Magne (1877-1944) \\ Henri-Marcel Magne (1877-1944)
}

\section{Vanessa Aspart}

\section{OpenEdition}

\section{Journals}

Édition électronique

URL : http://journals.openedition.org/lha/217

DOI : 10.4000/lha. 217

ISSN : 1960-5994

Éditeur

Association Livraisons d'histoire de l'architecture - LHA

Édition imprimée

Date de publication : 10 décembre 2009

Pagination : 11-20

ISSN : 1627-4970

Référence électronique

Vanessa Aspart, « Henri-Marcel Magne (1877-1944) », Livraisons de l'histoire de l'architecture [En ligne], 18 | 2009, mis en ligne le 10 décembre 2011, consulté le 01 mai 2019. URL : http:// journals.openedition.org/lha/217; DOI : 10.4000/lha.217

Ce document a été généré automatiquement le 1 mai 2019.

Tous droits réservés à l'Association LHA 


\title{
Henri-Marcel Magne (1877-1944)
}

\author{
Henri-Marcel Magne (1877-1944) \\ Henri-Marcel Magne (1877-1944)
}

Vanessa Aspart

1 Né en 1877 à Paris dans une famille d'architectes depuis trois générations, Henri-Marcel Magne, second enfant de Lucien Magne (1849-1916) et de Marie Geneviève Joséphine Lucile Le Verrier (1853-1931), est initié dès son plus jeune âge aux rudiments de l'architecture. Après des études au lycée Charlemagne, il est admis en juillet 1895 à l'École des beaux-arts. Sa scolarité, récompensée à maintes reprises par des médailles (mathématiques, perspective, dessin, modelage...) et le prix Müller-Soehnée, augure des débuts prometteurs. En 1899, Lucien Magne, alors titulaire de la chaire d'art appliqué aux métiers, le nomme préparateur de son cours au Conservatoire national des arts et métiers tandis qu'en 1900, ils collaborent dans le cadre de l'exposition universelle. Père et fils ne cesseront dès lors de s'associer, l'un consolidant et restaurant, l'autre fournissant des modèles de cartons de vitraux ou de mosaïques. Tenté pendant un temps par la peinture, Henri-Marcel étudie dans les ateliers de Luc-Olivier Merson (1846-1920), Albert Pierre René Maignan (1845-1908) et Alfred Paul Marie Panon Desbassayns de Richemond (1857-1911) et expose régulièrement des tableaux au salon des artistes français, notamment la Construction d'un viaduc (1909) et L'Architecte (1910). Mais ce penchant est contrarié par les projets de Lucien, qui souhaite voir son fils perpétuer les préceptes du cours d'art appliqué aux métiers, fondés sur la connaissance des caractéristiques de la matière travaillée. Homme de son temps, acquis aux théories du Conservatoire, HenriMarcel déploie son originalité à travers les chantiers religieux et civils. Bénéficiant du soutien paternel ainsi que d'appuis professionnels, il fournit cartons et modèles de vitraux selon les nécessités cultuelles de la capitale et les impératifs de reconstruction imposés par la Grande Guerre. 


\section{La décoration monumentale : l'influence des réseaux personnels et professionnels}

2 Le déroulement de sa carrière est intimement lié aux contacts établis par l'entremise de son père. En effet, sa production s'étend dans des zones géographiques bien définies, souvent dépendantes du champ d'action d'architectes en chef des monuments historiques alors en fonction. Trois rencontres s'avèrent déterminantes ${ }^{1}$. Héritiers de Charles-Louis Genuys (1852-1928) et de Lucien Magne et membres en 1926 de l'union syndicale des architectes français, Pierre Sardou (1873-1952), Bernard Haubold (1875- ?) et CharlesHenri Besnard (1881-1946), tous trois architectes en chef des monuments historiques, suivent des trajectoires qui s'entrecroisent parfois, au gré des chantiers. Ils collaborent, s'affrontent amicalement, entrent en concurrence. Pierre Sardou, élève de Lucien Magne, est le premier à requérir les compétences de son ami Henri-Marcel. Dans les années 1910, il fait appel à lui non seulement pour la décoration du préau d'une école maternelle parisienne, à l'angle des rues Gabriel-Vicaire, Dupetit-Thouars et Paul-Dubois, mais aussi pour celle de l'église Notre-Dame-du-Rosaire à Paris et du manoir Denouval à Andresy, propriété de Mme Hershey Marsh. Pour ces constructions destinées à des usages fort différents, Henri-Marcel s'adapte et répond aux attentes du bâtisseur : toiles marouflées sur le thème du labeur des gens de la terre et de l'océan pour l'école de la rue PaulDubois, triptyque sur L'éducation de la Vierge et modèles de vitraux religieux pour l'église Notre-Dame-du-Rosaire, carton de vitrail profane (Apollon et les Muses) et de mosaïques pour le manoir Denouval.

3 Bernard Haubold, autre élève de Lucien Magne, côtoie fréquemment Henri-Marcel sur les chantiers dès la fin de la première guerre mondiale. Leur rivalité en 1919 pour l'obtention de la chaire d'art appliqué aux métiers ne ternit en effet pas leurs relations: en 1919-1920, Haubold soutient le projet de monument aux Morts pour la collégiale SaintAubin de Guérande de son ancien concurrent face à celui de M.Vallet ${ }^{2}$, sculpteur à Nantes. Actif dans la Marne à partir des années 1920-1930, principalement dans les arrondissements de Châlons-sur-Marne et de Vitry-le-François, Haubold, chargé de restaurer les édifices dévastés durant la Grande Guerre, confie à Henri-Marcel la tâche de créer des modèles de vitraux dans pas moins de douze églises ${ }^{3}$, parmi lesquelles les églises de Caurel-les-Lavannes, Saint-Cyr-Sainte-Julitte à Cormicy et Saint-Souplet-sur-Py. Celuici y voit l'opportunité de mettre en œuvre sa doctrine relative au vitrail ${ }^{4}$ : verres teintés dans la masse, tout au plus gravés mais rejet des émaux et des verres martelés, opalisés, plissés, striés, dont l'usage doit être réservé aux constructions civiles, couleurs vives et chatoyantes, forte présence des plombs qui structurent la composition et dessinent les motifs, restriction de la grisaille. Á l'instar de son père, il refuse l'unité de style et «[fait] œuvre moderne dans des édifices anciens $»^{5}$. Une phrase résume bien sa pensée et ses réalisation : «Le choix d'un style n'est pas en question puisqu'il s'agit de créer une œuvre d'art [...]. Ce qui importe, c'est que le vitrail ne soit pas un tableau, que la composition s'accorde avec son cadre architectural, qu'elle demeure sur la surface et qu'elle l'enrichisse par une harmonie de couleurs sans effets de trompe-l'œil ${ }^{6} »$. 
III. 1 : La mort de Joseph, vitrail

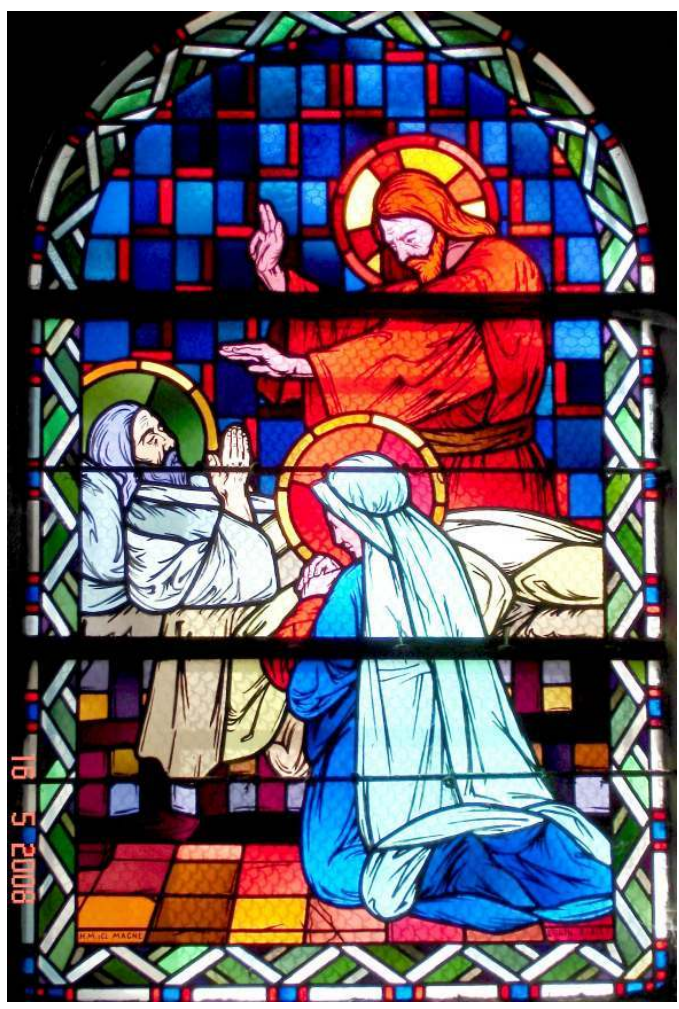

Henri-Marcel et Claude Magne (maquettes), ateliers Lorin (exécution), vers 1937-1939, bas-côté sud, église Saint-Cyr-Sainte-Julitte, Cormicy

Cl. Vanessa Aspart. 
III. 2 : L'onction des malades, vitrail

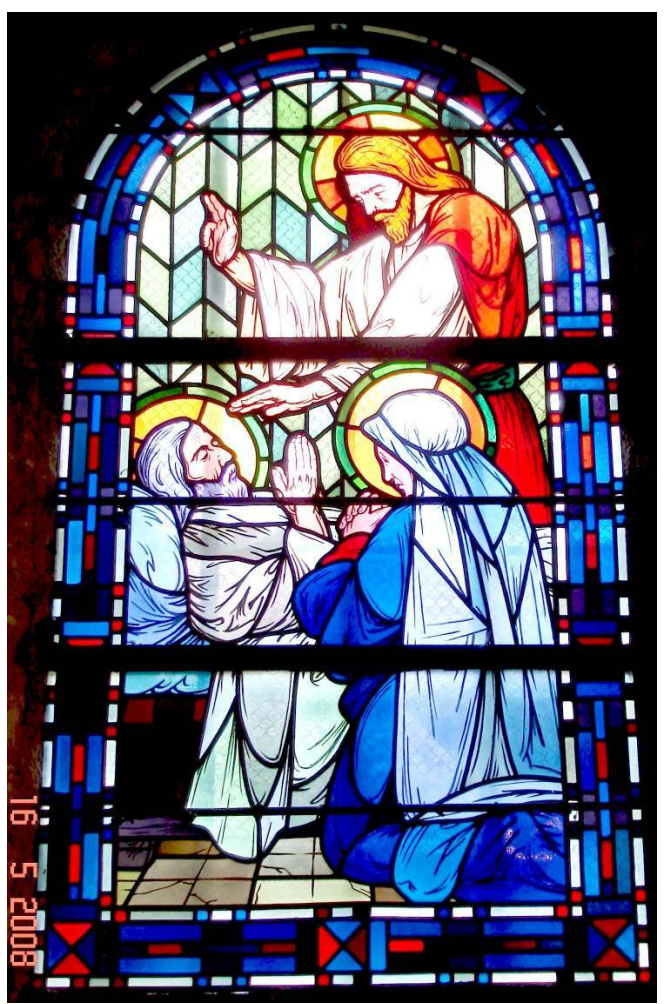

Henri-Marcel et Claude Magne (maquettes), ateliers Lorin (exécution), 1937-1939, bas-côté sud, église, Caurel-les-Lavannes.

Cl.Vanessa Aspart.

4 Cependant, sa méthode de travail, qui consiste à employer un même carton pour des sujets différents ou semblables, le conduit parfois à une certaine monotonie, qu'il tente de dissimuler en modifiant couleurs et motifs décoratifs : La mort de Joseph (ill. 1) devient ainsi l'Onction des malades' (ill. 2) tandis que le saint Michel de l'église de Saint-Souplet-sur$\mathrm{Py}^{8}$, dont la maquette est réalisée en 1932, est une transposition du saint Michel, signé et daté de 1929, de l'église Saint-Michel-des-Batignolles à Paris. Cette dernière, édifiée par Haubold à partir de 1912, présente en outre un remarquable chemin de croix, exécuté en 1937 dans divers bois coloniaux par A. Messager d'après des modèles d'Henri-Marcel, qui ont probablement inspiré le chemin de croix en pierre de l'église de Saint-Souplet-sur-Py (ill.3). 


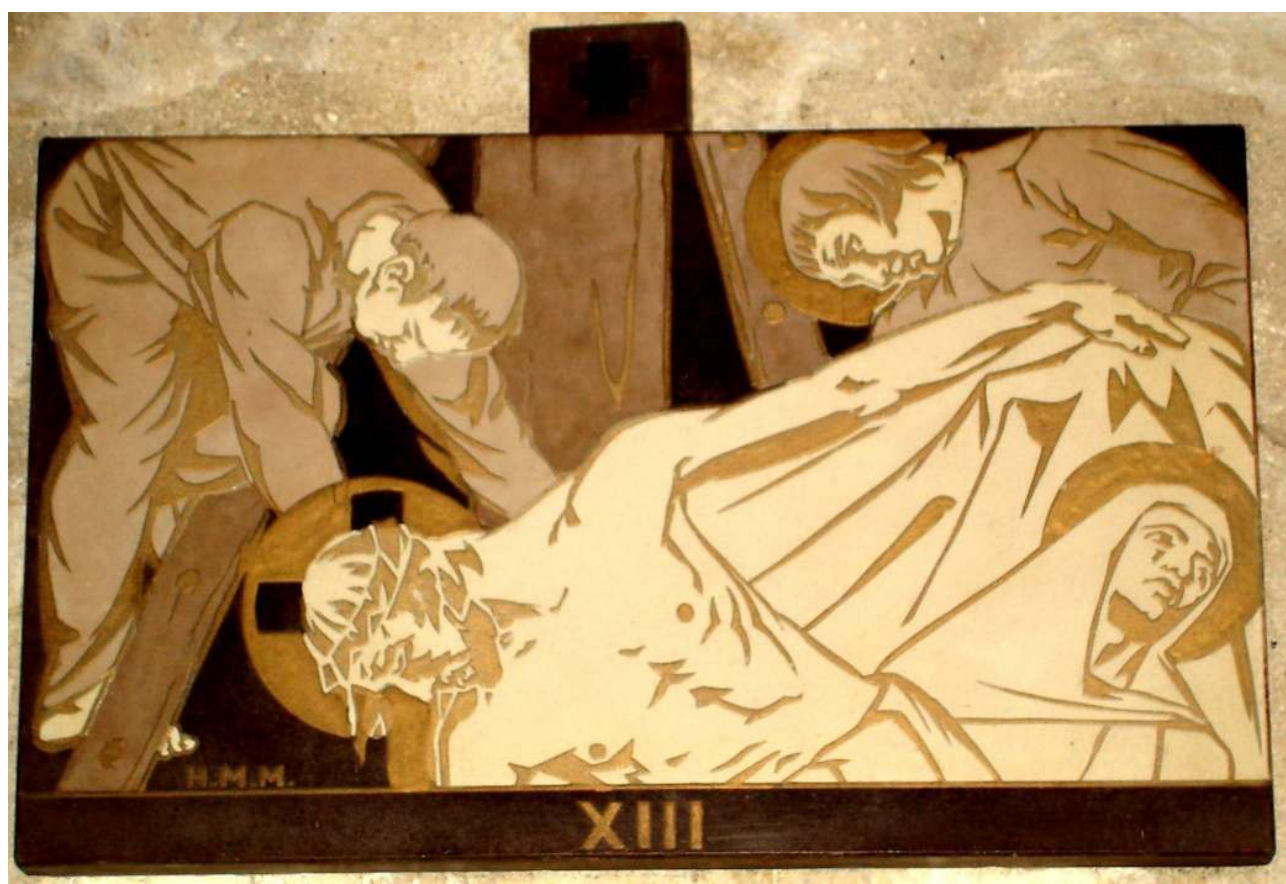

Pierre, date inconnue, église, Saint-Souplet-sur-Py

$\mathrm{Cl}$. Vanessa Aspart.

5 Henri-Marcel applique ainsi cette stratégie de remploi aussi bien au vitrail qu'à d'autres arts décoratifs, démarche qui lui confère une efficacité et une rapidité nécessaires au vu du nombre élevé d'églises à décorer durant l'entre-deux-guerres. L'audace de ces deux chemins de croix résident dans les choix d'Henri-Marcel: cadrage resserré sur les personnages représentés à mi-corps ou en buste, renouvellement et adaptation d'une technique sophistiquée, la marqueterie, d'ordinaire appliquée au mobilier, style graphique souligné par des aplats de couleur et une absence de profondeur due à l'arrière-plan noir. Ce style graphique à la ligne nerveuse caractérise également les peintures murales qu'il exécute en 1922 sur les murs extérieurs du presbytère de l'église Saint-Christophe-de-Javel ${ }^{9}$ à Paris. Les plans de cette église sont dessinés en 1921-1922 par Charles-Henri Besnard, inventeur avec l'ingénieur Julien-Pierre-Bertrand Bessoneau, d'un procédé de construction rapide dans lequel les éléments structurels composés de ciment armés sont moulés en série. Dans une perspective rationaliste, il met en œuvre ce procédé novateur à l'église Saint-Christophe-de-Javel tandis qu'Henri-Marcel, fervent défenseur du béton comme d'autres artistes de son époque, imagine une technique de peinture à base de ciment s'inspirant de la fresque pour habiller les murs du presbytère.

\section{Une utilisation expérimentale des matériaux}

6 Henri-Marcel, à travers ses écrits, alimente le débat qui a lieu autour d'un nouveau matériau de construction: le béton. La manière de l'employer ne fait pas l'unanimité parmi tous les architectes, notamment dans le domaine de l'architecture religieuse. Nombre de bâtisseurs conseillent de parer les murs de béton d'un revêtement tandis qu'Henri-Marcel s'élève contre la modalité de dissimulation d'un matériau, qui possède 
son esthétique propre ${ }^{10}$. Il appelle les architectes à multiplier les constructions en béton, en respectant les lieux dans lesquelles elles s'insèreront et les artistes, à décorer les surfaces de ces édifices, soit par moulage, comme pour le pavillon de la Société de l'art appliqué aux métiers érigée pour l'exposition des arts décoratifs de 1925 par Bernard Haubold et Charles-Henri Besnard ${ }^{11}$, soit en les recouvrant d'un enduit, peint à fresque ou décoré de sgraffite. Il est aussi possible d'envisager un décor de mosaïque, en plaçant celle-ci au fond des coffrages avant le pilonnage du béton ${ }^{12}$. Ainsi, les architectes peuvent tirer des effets artistiques du béton, tout comme des autres matériaux utilisés précédemment, "à condition qu'au lieu de se cantonner dans les formules d'art applicables à ces matériaux, on cherche des formules nouvelles, applicables à une matière nouvelle $»^{13}$.

\section{4 : Henri-Marcel Magne, Saint Christophe et l'automobiliste}

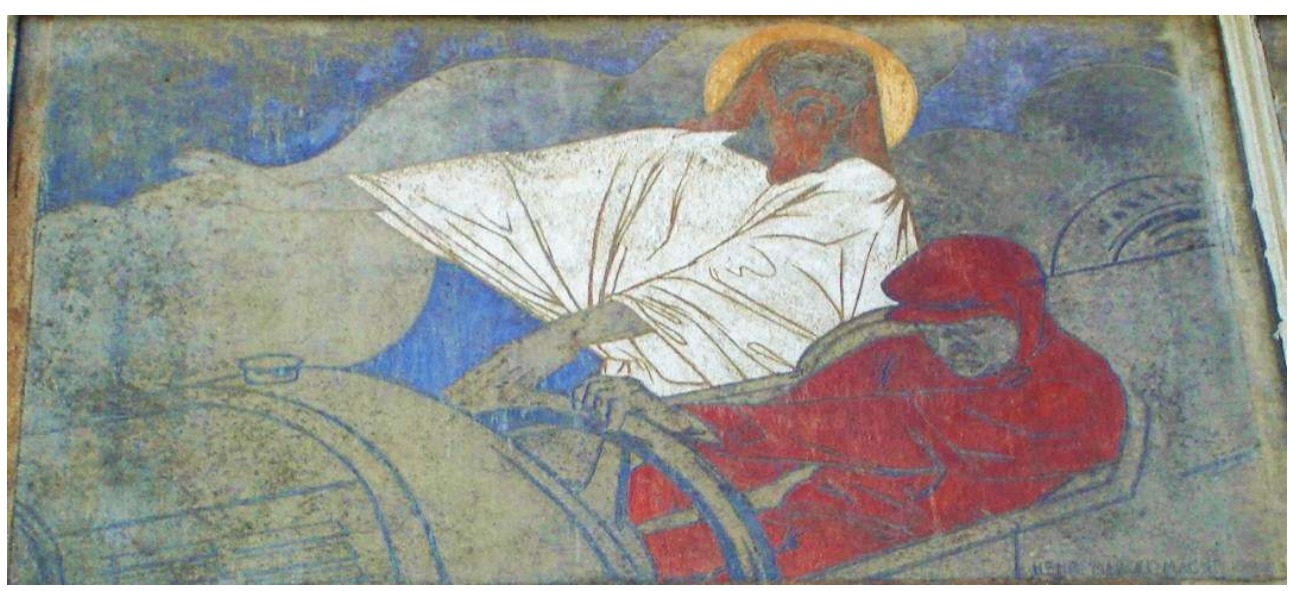

Plaque de ciment incisé et gravé, 1922, église Saint-Christophe-de-Javel, Paris

Cl. Vanessa Aspart.

Le décor du presbytère de l'église Saint-Christophe-de-Javel, représentant La légende de saint Christophe intercédant en faveur des voyageurs (ill. 4), répond bien à ce principe énoncé : devant l'emploi de plus en plus répandu du ciment, Henri-Marcel modifie la technique ancienne de la fresque en remplaçant le support de chaux fraîche par un support de ciment frais. Il effectue des recherches dans son laboratoire du conservatoire national des arts et métiers sur les pigments, qui doivent répondre à des critères de permanence et de pérennité, particulièrement face aux composants du ciment, afin d'éviter toute réaction physico-chimique. Les oxydes de fer, par exemple, semblent convenir à cette nouvelle technique ${ }^{14}$. Cependant, en mettant en œuvre ce procédé à base de ciment, Henri-Marcel limite le nombre de couleurs pouvant être utilisées et de nuances pouvant être créées, le ciment risquant de les assombrir. En outre, ces cinq plaques de ciment, par ailleurs incisé et gravé, présentent de graves lacunes. Certains pigments craignent en effet l'action de l'eau et des pluies acides, ainsi que le $\mathrm{pH}$ basique du ciment, ce problème de $\mathrm{pH}$ basique existant déjà dans la vraie fresque. Dans le cas présent, il semblerait que les pigments blanc et bleu soient ceux qui résistent le mieux aux conditions climatiques.

Ce test de peinture sur ciment frais n'est pas renouvelé à l'intérieur de l'église SaintChristophe-de-Javel, pour laquelle Henri-Marcel reçoit la commande supplémentaire d'orner le plafond de la nef centrale divisée en six travées et la voûte en forme de cul-de- 
four du sanctuaire. Il choisit en effet de peindre sur des plaques de plâtre mêlé de fils. Ce renouvellement de la fresque par le ciment semble demeurer une initiative isolée dans son œuvre. Il poursuit cependant son action et sa réflexion en faveur du béton en introduisant dans son cours au Conservatoire l'étude du béton armé et de ses applications artistiques.

Quelques années plus tard, Henri-Marcel, toujours intéressé par les matériaux et leurs caractéristiques physiques en vu d'applications artistiques, met au point deux nouveaux procédés de montage des vitraux afin de pallier le manque de matières premières, notamment de plomb, primordial pour rénover les verrières détruites pendant la seconde guerre mondiale. Après diverses tentatives, le conseil d'administration de l'institut de recherche et de coordination artistique et technique ${ }^{15}$, qui travaille en collaboration avec la société d'encouragement à l'industrie nationale, créée en 1801 et dont Henri-Marcel Magne assure la présidence depuis 1939, retient, le 20 février 1941, un prototype en aluminium de $1 \mathrm{~mm}$ d'épaisseur (ill. 5).

III. 5 : Henri-Marcel Magne, montage de vitraux en aluminium, schéma.

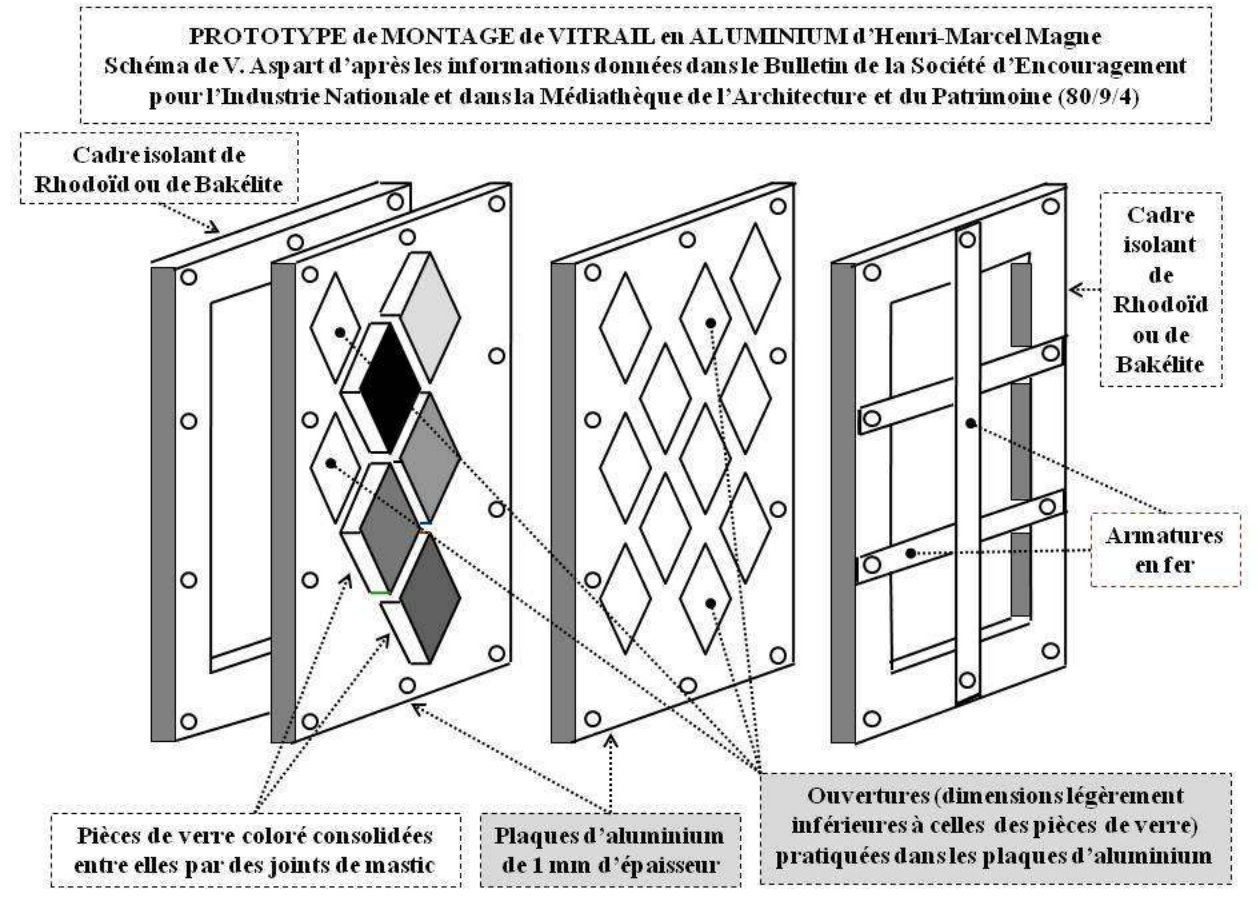

Cl.Vanessa Aspart.

Dans ce procédé ${ }^{16}$, deux plaques d'aluminium pur sont découpées, repercées et traitées à l'aide d'une solution bouillante de chromate légèrement carbonaté, suivie d'un dépôt général de vernis. Cette phase de traitement s'intitule la "protalisation ». Une des deux plaques est retournée sur son envers. Les pièces de verre sont disposées sur cette plaque posée à l'envers et sont assemblées entre elles par une cloison de mastic. L'ensemble est recouvert de la deuxième plaque protalisée et les deux plaques sont maintenues entre elles par des vis et des écrous d'aluminium, qui évitent ainsi tout mécanisme galvanique. L'opération prend fin avec le masticage et le contre-masticage habituels. Ce procédé paraît avantageux sur plusieurs points : protection des plaques d'aluminium grâce à la protalisation, simplicité du montage, légèreté des panneaux réalisés avec ce système de 
montage, facilité de remplacement des pièces usagées. Il existe cependant des inconvénients. Il faut en effet prendre des précautions lors du montage des vitraux, notamment éviter le contact entre l'aluminium des plaques et le fer des armatures, susceptibles de rouiller, soit en réalisant les armatures en fer parkésiré, soit en encadrant le panneau d'un matériau plastique isolant tel que le rhodoïd ou la bakélite. En outre, ce procédé de montage présente un coût non négligeable, comprenant le prix d'acquisition de l'outillage spécifique au découpage et au percement des plaques de métal et le prix de revient qui est presque deux fois plus élevé dans le cas de plaques d'aluminium de $2 \mathrm{~mm}$ d'épaisseur (650 francs pour un mètre carré, 550 francs le mètre carré pour des plaques d'aluminium de $1 \mathrm{~mm}$ d'épaisseur au lieu de 350 francs dans le cas d'un mètre carré de vitrail comportant quatre cents pièces montées en plomb). Enfin, il faut s'assurer de l'étanchéité des cloisons de mastic qui enserrent les verres, cette étanchéité étant habituellement réalisée par les plombs et réfléchir à une solution qui puisse permettre d'adapter le vitrail ainsi monté, lequel présente un cadre d'isolement en plastique, aux ferrures existantes. Malgré ces points négatifs, la Commission est favorable à un essai dans un monument. La même année, Henri-Marcel, qui effectue également des expérimentations sur les matières plastiques, soumet à l'institut de recherche et de coordination artistique et technique (I.R.C.A.T.), dans la séance du 25 avril 1941, un panneau exécuté selon ce nouveau procédé. Ce procédé nécessite un profil spécial pour les cloisons de montage afin d'éviter la flexion perpendiculaire au plan du vitrail. «Les éléments sont réunis aux points de jonction et soudés, de même que les attaches des vergettes, au rhodoïd dissous dans l'acétone ${ }^{17}$." Les avantages suivants séduisent la Commission: "Résistance et légèreté, facilité d'exécution des pièces de dimensions grandes et moyennes à contours droits ou simple courbure, facilité et solidité de la soudure, possibilité de repiquer une pièce en sectionnant le nombre de cloisons nécessaires pour l'atteindre et en les ressoudant après remplacement de la pièce, facilité de souder des tringles de renfort ${ }^{18}$. ». Ce type de montage est donc très adapté à des verrières à dessins géométriques ou de grandes dimensions. De plus, les tests effectués sur la résistance du matériau à l'humidité, aux fortes chaleurs et au gel se sont révélés très satisfaisants. Quant au coût de revient, il sera peu élevé en comparaison de celui d'un montage en plomb grâce à la fabrication industrielle en série. Mais la Commission émet cependant quelques réserves: elle préfèrerait consacrer ce procédé à des verrières entièrement neuves, plutôt qu'à la restauration de vitraux anciens. En conséquence, elle propose d'effectuer un premier essai dans une des églises en construction dans la région parisienne. Mais les archives de la médiathèque ne mentionnent pas l'église qui bénéficiera de cette nouvelle technique et il semblerait qu'en 1942, ces deux procédés de montage, en aluminium et en rhodoïd, n'aient pas encore été appliqués ${ }^{19}$.

11 Cette expérience reste par ailleurs sans suite : Henri-Marcel Magne décède en effet le $1^{\text {er }}$ juillet 1944 à Champagne-sur-Seine, avant d'avoir pu achever le carton de vitrail destiné à l'église Saint-Martin-des-Champs ${ }^{20}$. Il est enterré à Eaubonne, dans le caveau familial.

Malgré sa formation d'architecte, Henri-Marcel Magne ne construit aucun édifice. Par le jeu des réseaux professionnels et amicaux, tissés lors de son apprentissage sur les chantiers de restauration de son père, il obtient essentiellement des commandes de modèles de vitraux pour des églises classées ou en cours d'édification. Titulaire, à la mort de son père, de la chaire d'art appliqué au conservatoire national des arts et métiers, il œuvre en faveur de l'union de l'art et de l'industrie et teste dans son laboratoire les propriétés de matériaux tels que le ciment, l'aluminium et le plastique, contribuant par 
ses recherches au renouvellement de la peinture murale au XXe siècle ainsi qu'à une réflexion sur de nouvelles formules de montage des vitraux.

\section{NOTES}

1. . Voir Les Concours des monuments historiques: de 1883 à 1979, cat. exp., Paris, caisse nationale des monuments historiques et des sites, 7 octobre 1981 - 31 janvier 1982, Paris, caisse nationale des monuments historiques et des sites, 1981, $128 \mathrm{p}$.

2. . Arch. de la médiathèque de l'architecture et du patrimoine, collégiale Saint-Aubin de Guérande, 0081/044/0014.

3. . Henri-Marcel décore également cinq autres églises dans la Marne, sous la direction de l'inspecteur des monuments historiques Eugène Rattier (1864-1947). Sur quarante édifices de la Marne bénéficiant de la pose de nouveaux vitraux, Henri-Marcel travaille donc pour dix-sept d'entre deux.

4. . Lucien et Henri-Marcel Magne. L'Art appliqué aux métiers. Décor du verre : gobeleterie, mosaïque, vitrail. Paris, H. Laurens, $2^{\mathrm{e}}$ éd. complétée, 1927, t. III, 228 p.

5. . Henri-Marcel Magne, L'Architecture, Paris, F. Rieder, 1922, p. 111.

6. . Henri-Marcel Magne, L'Art appliqué aux métiers, op. cit., p. 191.

7. . Les deux maquettes correspondantes sont présentées par B. Haubold au directeur général des beaux-arts le 27 novembre 1937. Voir arch. de la médiathèque de l'architecture et du patrimoine, 0081/051/ 0021.

8. . Voir arch. de la médiathèque de l'architecture et du patrimoine, 0081/051/0188.

9. . Voir Charles-Henri Besnard. L'Église Saint-Christophe-de-Javel, description raisonnée, Paris, Imp. de P. Masch, L. Seitz, 1930, 55p.

10. . Voir Henri-Marcel Magne, "L'architecture et les matériaux nouveaux ", Art et décoration, juillet 1914-décembre 1919, tome XXXVI, p.85-96 et Henri-Marcel Magne, « Les tendances de l'architecture religieuse moderne ", La Renaissance de l'Art français, janvier 1929, p. 20-25.

11. . Henri-Marcel Magne réalise notamment des modèles pour une partie du mobilier de ce pavillon et compose la salle à manger.

12. . Voir Henri-Marcel Magne, Rapport sur la convenance de la décoration du béton armé par l'utilisation de la matière même, congrès technique international de la maçonnerie et du bétonnement, Rouen, Imp. Wolf, 1928, 10 p.

13. . Ibid., p. 9.

14. . Yvanhoé Rambosson, "Le presbytère de l'église Saint-Christophe à Paris ", L'Illustration, 19 juillet 1924, p. 61.

15. . L'Institut de Recherche et de Coordination Artistique et Technique (I.R.C.A.T.) existe depuis le le 10 novembre 1937 et a été fondé par le sous-secrétariat d'Etat à l'enseignement technique. Son contrôle est assuré par la double direction des beaux-arts et de l'enseignement technique.

16. . Voir le Bulletin de la société d'encouragement pour l'industrie nationale, juin-juillet 1939, août-septembre 1939, juillet-septembre 1941, octobre-décembre 1941 et arch. de la médiathèque de l'architecture et du patrimoine, 80/9/4. 
17. . Arch. de la médiathèque de l'architecture et du patrimoine, op. cit., rapport à la commission par M. Huignard, comité consultatif d'architecture, séance du 21 juillet 1941.

18. . Bulletin de la société d'encouragement pour l'industrie nationale. 1941, op. cit., p. 349.

19. . Arch. nat., $\mathrm{F}^{21} 6987$ : une lettre d'Henri-Marcel Magne adressée au secrétaire général des beaux-arts et datée du 30 mai 1942, permet d'envisager cette hypothèse.

20. . Arch. nat., $\mathrm{F}^{21} 6841$.

\section{RÉSUMÉS}

Descendant d'architectes, la carrière d'Henri-Marcel Magne semble tracée. Après une formation académique à l'École des beaux-arts dans la section architecture, il s'oriente vers la peinture, en fréquentant les ateliers de Luc-Olivier Merson, d'Albert Pierre René Maignan et d'Alfred Paul Marie Panon Desbassayns de Richemond. Après sa participation au salon des artistes français pendant une dizaine d'années, il se tourne définitivement vers la décoration monumentale en réalisant peintures murales, cartons et maquettes de vitraux pour des édifices civils et religieux. Ses liens amicaux et professionnels parmi les architectes en chef des monuments historiques le conduisent à travailler essentiellement en Ile-de-France et dans la Marne. Professeur au conservatoire national des arts et métiers, il poursuit l'œuvre de son père au sein de cet établissement et favorise le rapprochement entre industriels, artistes et artisans. Dans son cours, il associe une connaissance pointue des matières à disposition des artistes, trop souvent négligée selon lui, à une application pratique et étudie lui-même au sein du laboratoire du conservatoire des matériaux tels que le béton, l'aluminium et le plastique.

Descending from architects, Henri-Marcel's career seems written. After an academic training in the Ecole des beaux-arts, in the department of architecture, he turns to painting and frequents the workshops of Luc-Olivier Merson, Albert Pierre René Maignan and Alfred Paul Marie Panon Desbassayns de Richemond. After his contribution to French Artists' exhibitions for approximately ten years, he turns definitely to monumental decoration by realizing mural paintings, cardboards and models of stained glasses windows for civil and religious buildings. His friendly and professional links among the chief architects of historical monuments leads him to work essentially in Ile-de-France and in Marne. Professor to CNAM, he prolongs his father's work within this establishment and facilitates the links between industrialists, artists and craftsmen. In the lessons he teaches, he always associates a precise knowledge of materials for artists' disposal, too often neglected according to him, to a practical application, and studiesfor his part, within the CNAM's laboratory, materials such as concrete, aluminum and plastic.

Als Sprössling der Architektenfamilie Magne standen Henri-Marcel die Türen zur Karriere weit offen.. Nach einer akademischen Ausbildung im Fach Architektur wendete er sich der Malerei zu in den Ateliers von Luc-Olivier Merson, Albert Pierre René Maignan und Alfred Paul Marie Panon Desbassayns de Richemond. Etwa zehn Jahre lang beteiligte er sich am Salon des artistes français, bevor er sich schließlich zur monumentalen dekorativen Kunst wendete, indem er Wandmalereien, Kartons und Modelle für Glasfenster in öffentlichen und religiösen Gebäuden entwarf. Dank seiner freundschaftlichen und beruflichen Beziehungen $\mathrm{zu}$ Architekten für Denkmalpflege durfte er hauptsächlich in der Île de France und in dem Département Marne arbeiten. Er wurde andererseits Lehrer an der Pariser CNAM Hochschule, wo er das Werk seines 
Vaters weiterführte und dort die Annäherung zwischen Unternehmern, Künstlern und Handwerkern bevorzugte. In seinem Unterricht bestand er auf das seiner Meinung nach bisher vernachlässigte Erwerben spezieller Kenntnisse über die Materialien, die den Künstlern zur Verfügung standen, was unbedingt mit der praktischen Anwendung verbunden werden sollte. Im Labor des CNAM untersuchte er selber Materialien wie Beton, Aluminium und Plastik.

\section{AUTEUR}

\section{VANESSA ASPART}

Vanessa Aspart est née en 1983. Diplômée de l'École du Louvre (2008), elle a soutenu en 2007 un mémoire de master 1 portant sur « les techniques de la peinture murale au XIX ${ }^{\mathrm{e}}$ siècle : supports, pigments, liants ", sous la direction d'Isabelle Pallot-Frossard. Suite à ce mémoire, elle a participé aux journées du Patrimoine, en présentant la technique de la peinture à la cire à l'église SaintVincent-de-Paul à Paris. En 2008, dans le cadre de son master 2, elle a mené des recherches sur la carrière d'Henri-Marcel Magne (1877-1944), sous la direction de Daniel Imbert et Alice Massé. Elle a rédigé un article sur cet artiste, qui est en cours de publication dans la revue Le Point Riche. Elle coordonne actuellement, pour les éditions du Louvre, un projet d'ouvrage consacré à la collection Rothschild. Adresse électronique : vanessa.aspart@gmail.com. 